\title{
Evaluation of lymphoma patients after hematopoetic stem cell transplantation in terms of early period cardiotoxicity development
}

\author{
Sedat Çelikçi, Vildan Özkocaman²๑, Fatih $\operatorname{Yaman}^{3} \odot$, Fahir Özkalemkaş \\ ${ }^{1}$ Department of Internal Medicine, Bursa Kestel State Hospital, Bursa, Turkey; ${ }^{2}$ Department of Hematology, Bursa Uludağ University \\ School of Medicine, Bursa, Turkey; ${ }^{3}$ Department of Hematology, Eskişehir Osmangazi University School of Medicine, Eskişehir, Turkey
}

\begin{abstract}
Objectives: Autologous hematopoietic stem cell transplant (AHSCT) following high-dose chemotherapy in recurrent lymphomas has become the standard treatment. However, this method leads to various toxic side effects, including cardiotoxicity. This study aims to determine the factors that may cause post-transplant cardiotoxicity.

Methods: A total of 35 patients older than 18 years old, diagnosed with recurrent lymphoma, who underwent AHSCT at the Uludağ University Hematology Department, were included in the study. The patients were evaluated in two groups, with and without cardiotoxicity after AHSCT. We separated the frequency of cardiotoxicity that developed during hospitalization after transplantation and patients who developed and did not develop cardiotoxicity. We compare some parameters including gender, age, lymphoma type, stage, cardiac risk factors before transplantation, the number of chemotherapy cycles and the use of rituximab before the transplantation, radiotherapy before transplantation, Karnofsky performance scale, the amount of Dimethyl sulfoxide among these patients. Also, we evaluated patients with echocardiopraphy before transplantation and measured left ventricle ejection fraction (LVEF). We use CTCAE V 4.0 system for evaluating cardiotoxicity level from Grade I to Grade V.

Results: Nine patients developed cardiac events. One patient developed Grade V MI and died despite treatment. Other eight toxicities developed Grade III-IV and returned by treatment. In terms of risk factors, LVEF of the group with cardiotoxicity was found to be significantly low $(p<0.05)$. There is no statistical difference between the two groups with other parameters.

Conclusions: Cardiotoxicity is a frequent complication of autologous stem cell transplantation. A detailed pretransplantation evaluation of all patents in terms of cardiac functions is essential to reduce cardiac morbidity. Therefore, patients should be evaluated cardiologically before transplantation and closely monitored for posttransplantation cardiac side effects. Some studies show that enalapril and carvedilol may be beneficial to prevent from cardiotoxicity. Although not in our routine, if approved by larger studies, it may be necessary to use agents such as enalapril and carvedilol in prophylaxis in reduce cardiotoxicity.

Keywords: stem cell transplantation, cardiotoxicity, lymphoma, chemotherapy
\end{abstract}


$\mathrm{L}$ ymphomas are heterogeneous groups of hematological malignancies. Lymphomas result from various differentiation stages of lymphocytes and show different immunological and clinical manifestations. They constitute $3-4 \%$ of all cancers seen worldwide [1]. Despite the high treatment success rate, there is significant resistance to treatment or recurrence. Generally, autologous hematopoietic stem cell transplantation (AHSCT) following high-dose chemotherapy is the standard treatment regimen in all patients with recurrent lymphoma [2]. This treatment process can cause various toxic effects, including cardiotoxicity. Congestive heart failure or pericarditis at a late stage is the causes of morbidity and mortality due to radiotherapy or treatment regimens involving cardiotoxic chemotherapeutic agents [3, 4]. Acute cardiac toxicities after AHSCT include cyclophosphamide-induced acute cardiomyopathy or hemorrhagic pericarditis, acute myocarditis, and bradycardia associated with dimethyl sulfoxide (DMSO) used to protect progenitor cells, cyclophosphamide regimens [4-6].

The most frequently used chemotherapy regimen in AHSCT lymphoma is the BEAM (melphalan, cytosine arabinoside, etoposide, carmustine) protocol. Drug toxicities are briefly mentioned in various articles and each of these drugs are used for the treatment of different patient groups with other protocols. However, there are not enough publications evaluating the frequency of cardiotoxicity development (incidence, risk factors, cardiac toxicity results) clinically in patients with recurrent lymphoma with autologous hematopoietic stem cell transplantation using the BEAM protocol. This study aims to determine the factors that may cause post-transplant cardiotoxicity.

\section{METHODS}

A total of 35 patients older than 18 years old, diagnosed with recurrent lymphoma under AHSCT at the Bone Marrow Transplant Unit of Uludağ University School of Medicine, Hematology Department, between January 2009 and October 2015 were included in the study. The data of the patients were retrospectively analyzed for the research protocol.

We planned to screen some factors associated with cardiac risk through the automation system of the hospital. These factors include cardiac risk factors (hy- pertension, diabetes mellitus, hypercholesterolemia, obesity, family history of cardiovascular disease, smoking) pre-transplant echocardiography, electrocardiography (ECG), blood tests (Creatine kinase, Creatine kinase kinase-mb, troponin-I) [6].

Cardiotoxicites were evaluated according to the Common Terminology Criteria for Adverse Events Version 4.0 system and toxicities were classified in severity from grade I to grade V. Grade I is asymptomatic, grade II does not require urgent treatment, grade III is symptomatic, grade IV represents lifethreatening case, grade $\mathrm{V}$ is death of all patients [7].

We designed this study to evaluate cardiotoxicity in early period after transplantation (during hospitalization). The patients were separated into two groups; the ones who developed cardiotoxicity after AHSCT and those who did not. Each group was compared in terms of some factors including gender, age, diagnosis, lymphoma, pre-transplant cardiac risk factors, chemotherapy cycles, using of rituximab, radiotherapy before transplantation, the karnofsky performance scale [8], the amount of DMSO, and the left ventricular ejection fraction (LVEF) values.

We use the BEAM (BCNU $300 \mathrm{mg} / \mathrm{m}^{2}$, etoposide $800 \mathrm{mg} / \mathrm{m}^{2}$, ARA-C, $1600 \mathrm{mg} / \mathrm{m} 2$, melphalan 140 $\mathrm{mg} / \mathrm{m}^{2}$ ) protocol as a standard chemotherapy regimen for patients with recurrent lymphoma [9].

DMSO is used in transplantation because it is a cryoprotective agent. We compared DMSO cassette numbers to see if there was a relationship with posttransplant cardiotoxicity.

Our study was approved by the Ethics Committee of Uludağ University Non-Interventional Clinical Studies on 16.02.2016 with the decision number 2016$3 / 7$.

\section{Statistical Analysis}

Statistical Package for the Social Science (SPSS13.0) program was used for statistical analysis of the data. The data were investigated using visual (histograms, probability plots) and analytical methods (Kolmogorov-Smirnov/Shapiro-Wilk's tests) to determine their normal distribution. Differences between groups were evaluated using Student's t-test for parametric data and the Mann-Whitney U-test for nonparametric data. Relationships between categorical variables were analyzed using a chi-squared test. Data were expressed as frequency and percentage, mean 
and standard deviation, median, minimum and maximum values. It was considered statistically significant when the $\mathrm{p}$ value was less than 0.05 .

\section{RESULTS}

The characteristics of the patients were presented in

Table 1. Patient characteristics

\begin{tabular}{|c|c|}
\hline & $\mathbf{n}=\mathbf{3 5}$ \\
\hline Age (years), median (range) & $46(22-65)$ \\
\hline \multicolumn{2}{|l|}{ Gender, n (\%) } \\
\hline Male & $19(54)$ \\
\hline Female & $16(46)$ \\
\hline \multicolumn{2}{|l|}{ Pre-transplant diagnosis, n (\%) } \\
\hline Non-Hodgkin Lymphoma & $23(66)$ \\
\hline Hodgkin Lymphoma & $12(34)$ \\
\hline \multicolumn{2}{|l|}{ Lymphoma stage, n (\%) } \\
\hline Stage I-II & $13(37)$ \\
\hline Stage III-IV & $22(63)$ \\
\hline \multicolumn{2}{|l|}{ Cardiovascular comorbidity, n (\%) } \\
\hline Yes & $12(34)$ \\
\hline No & $23(66)$ \\
\hline \multicolumn{2}{|l|}{ Pre-transplant rituximab using, n (\%) } \\
\hline Yes & $20(43)$ \\
\hline No & $15(57)$ \\
\hline $\begin{array}{l}\text { Number of chemotherapy cycle, } \\
\text { median (range) }\end{array}$ & $10(4-15)$ \\
\hline \multicolumn{2}{|l|}{ Pre-transplant radiotherapy, n (\%) } \\
\hline Yes & $6(17)$ \\
\hline No & $29(83)$ \\
\hline \multicolumn{2}{|l|}{ Karnofsky Performance Scale, n (\%) } \\
\hline 100 & $11(31)$ \\
\hline 90 & $23(66)$ \\
\hline 80 & $1(3)$ \\
\hline $\begin{array}{l}\text { Left ventricular ejection fraction (\%), } \\
\text { median (range) }\end{array}$ & $63(56-74)$ \\
\hline $\begin{array}{l}\text { Hospitalization time (days), } \\
\text { median (range) }\end{array}$ & $28(11-53)$ \\
\hline $\begin{array}{l}\text { Number of Cassette* (Stem cell } \\
\text { aggregated product), median (range) }\end{array}$ & $3(1-8)$ \\
\hline
\end{tabular}

*Cassette: DMSO + autologous plasma + Stem cell product
Table 1 . There were 35 patients, 19 of whom (54\%) were male and 16 of whom $(46 \%)$ were female. The median age of the patients was 46 , the youngest patient was 22 while the oldest was 65 .

Nine $(26 \%)$ of the patients developed cardiotoxicity after transplantation. Post-transplant cardiotoxicities are summarized in Table 2.

We divided the patients into two groups: those who developed cardiotoxicity after transplantation (n $=9)$ and those who did not $(n=26)$. The group which didn't experience cardiac events is named control group. We found that the median age was 52 in the group with cardiotoxicity and 46.5 in the control group. There was no statistically significant difference in terms of age factor between the two groups ( $p=$ $0.661)$. When we analyzed the two groups in terms of gender distribution, there were 2 males, 7 females in the group of cardiotoxicity and 17 males and 9 females in the control group. No significant difference was found between the two groups in terms of gender $(p=$ $0.050)$, either. When two groups were examined with respect to diagnosis, there were $6 \mathrm{NHL}, 3 \mathrm{HL}$ in the group of cardiotoxicity, 17 NHL and 9 HL in the control group. There was also no significant difference between the two groups related to the diagnosis of the disease $(p=1.000)$.

When lymphoma stages were evaluated as stage I, II, III, IV, a statistically significant difference was found between the group of cardiotoxicity and the control group $(p=0.047)$. It was determined that $5(56 \%)$ of the patients who developed cardiotoxicity had stage IV lymphoma and 4 (44\%) of them had stage II lym-

\section{Table 2. Post-transplant cardiotoxicities}

\begin{tabular}{lcc}
\hline $\begin{array}{l}\text { Number of patients who developed } \\
\text { cardiac events after transplantation }\end{array}$ & $\mathbf{n = 9}$ & $\mathbf{\%}$ \\
\hline Rhythm Disorders & & \\
$\quad$ HVRAF & 3 & 33 \\
\hline Sinus tachycardia & 2 & 22 \\
\hline Bradycardia & 1 & 11 \\
\hline VT, VF & 1 & 11 \\
$\quad$ Atrial extrasystole, atrial fibrillation & 1 & 11 \\
\hline Ischemic Events & & \\
\hline$\quad$ Myocardial infarction & 1 & 11 \\
\hline
\end{tabular}

HVRAF: higher ventricular rate atrial fibrillation,

VT: ventricular tachycardia, VF: ventricular fibrillation 
phoma. However, in terms of stage IV lymphoma, no significant difference was found concerning the development of cardiotoxicity in advanced stage patients compared to stage I-II-III lymphoma patients ( $p=$ 0.103 ). When examined in terms of cardiovascular comorbidities before transplantation, there was no significant difference in both groups. In the group of cardiotoxicity, comorbid disease was detected in only $1(11 \%)$ patient, while in the control group, $11(42 \%)$ patients had cardiac comorbid disease before transplantation. It was observed that pre-transplant using of rituximab in chemotherapy protocols did not make a statistically significant difference in both groups ( $p$ $=0.700)$. There were $6(67 \%)$ patients in group of cardiotoxicity and 14 (54\%) patients in the control group related to use of rituximab in pre-transplantation period. It was found that the number of chemotherapy cycles before transplantation did not make a statistically significant difference between the two groups ( $p$ $=0.590)$. Also there wasn't a significant difference be- tween the two groups related to pre-transplant radiotherapy $(p=0.635)$. Two $(22 \%)$ patients in the cardiotoxic group and $4(15 \%)$ patients in the control group received pre-transplant radiotherapy.

It was observed that Karnofsky performance score (KPS) of the patients before AHSCT did not make a significant difference in the two groups $(p=0.083)$. It was determined that $1(11 \%)$ of the patients had 100 KPS, 7 (78\%) of them had KPS 901 (11\%) of them had KPS 80 in the group of cardiotoxicity.

When the LVEF values calculated during the routine echocardiography performed before transplantation were compared, a significant difference was found between the two groups $(p=0.039)$. In the group of cardiotoxicity, the median LVEF value was 60, minimum 58 and maximum 65, while the median SVEF value was 65 , minimum 56 and maximum 74 in the control group. When both groups were evaluated in terms of duration of hospitalization, there was no significant difference among them $(p=0.810)$. In

Table 3. Evaluation of patients with lymphoma who developed cardiotoxicity after AHSCT in terms of risk factors

\begin{tabular}{|c|c|c|c|}
\hline & $\begin{array}{l}\text { Cardiotoxicity } \\
\quad(n=9)\end{array}$ & $\begin{array}{l}\text { Control } \\
(n=26)\end{array}$ & p value \\
\hline Age (years), median (range) & $52(23-64)$ & $46.5(22-65)$ & 0.661 \\
\hline Male/female & $2 / 7$ & $17 / 9$ & 0.050 \\
\hline NHL/HL & $6 / 3$ & $17 / 9$ & 1.000 \\
\hline Stage & & & 0.047 \\
\hline Stage I & $0(0 \%)$ & $1(4 \%)$ & \\
\hline Stage II & $4(44 \%)$ & $8(31 \%)$ & \\
\hline Stage III & $0(0 \%)$ & $11(42 \%)$ & \\
\hline Stage IV & $5(56 \%)$ & $6(23 \%)$ & \\
\hline Comorbidity & $1(11 \%)$ & $11(42 \%)$ & 0.121 \\
\hline Rituximab using & $6(67 \%)$ & $14(54 \%)$ & 0.700 \\
\hline Number of chemotherapy cycle & $11(6-13)$ & $11(4-15)$ & 0.590 \\
\hline Pre-transplant radiotherapy & $2(22 \%)$ & $4(15 \%)$ & 0.635 \\
\hline Karnofsky performance scale & & & 0.083 \\
\hline 100 & $1(11 \%)$ & $10(38.5 \%)$ & \\
\hline 90 & $7(78 \%)$ & $16(61.5 \%)$ & \\
\hline 80 & $1(11 \%)$ & $0(0 \%)$ & \\
\hline LVEF (\%), median (range) & $60(58-65)$ & $65(56-74)$ & $\mathbf{0 . 0 3 9}$ \\
\hline Period of hospitalization (days), median (range) & $30(11-38)$ & $27.5(21-53)$ & 0.810 \\
\hline Number of cassettes DMSO, median (range) & $3(1-6)$ & $2(1-8)$ & 0.342 \\
\hline
\end{tabular}


the group of cardiotoxicity, the median period of hospitalization is 30 days, at least 11 and at most 38 days. The median duration of hospitalization was found to be 27.5 days in the control group, with a minimum of 21 days and a maximum of 53 days. The number of cassettes infused during the transplant we used to indirectly measure toxicity of DMSO did not make a significant difference in either group $(p=0.342)$. The median value of the number of cassettes in the group with cardiotoxicity was found to be 3 , minimum 1 and maximum 6. Considering the control group, the median value of the number of cassettes in this group was found to be 2, minimum 1 and maximum 8 cassettes.

Evaluation of patients with lymphoma who developed cardiotoxicity after AHSCT in terms of risk factors is summarized in Table 3.

\section{DISCUSSION}

AHSCT is used all over the world for recurrent lymphomas after the implementation of the standard chemotherapy and radiotherapy regimens [10]. Although this group of patients, whose number is increasing day by day, can develop a wide range of events especially early cardiac events may be an important cause of mortality and morbidity. It also requires urgent intervention [11]. In this single-center study, we tried to determine the characteristics, incidence, and risk factors of cardiotoxicities that occur in the post-transplant hospitalization process of the patients diagnosed with lymphoma.

When we review the literature on the frequency of cardiac events developing after transplantation, in the study of the first 100-day post-transplant study involving 2821 patients, published by Murdych and Weisdorf [12] in 2001, 26 (0.9\%) patients developed major cardiac events and 13 were found to be mortal. In a study in which 249 patients with transplantation with NHL were evaluated retrospectively, 30 (13\%) patients developed supraventricular tachycardia [13]. Also, it was mentioned in this study that patients with lymphoma developed more cardiac events after autologous transplantation than allogeneic transplantation. The cardiac event ratio of patients with allogeneic transplantation is $0.9 \%$ after transplantation. On the other hand, patients with autologous transplantation have higher risks of cardiac complications in compar- ison with allogeneic transplantation. Therefore, patients with lymphoma under autologous transplantation are very important in terms of cardiac events among patients with transplantation.

The cardiac events we observed during our study were frequently caused by rhythm disorders. MI was detected in only one patient. Especially the most frequently observed rhythm disorder appeared to be AF. In the study conducted by Hidalgo et al. [13], AF was found to be $91 \%$ among the supraventricular tachyarrhythmias. No patient developed heart failure in the early period. There are studies indicating that cardiotoxicity is observed as heart failure especially in the late period after transplantation [11, 14].

When we defined the patients as stage I-II-III-IV, we observed that there was a difference among these groups in terms of post-transplant cardiac event development. When we performed subgroup analysis, it was observed that patients in the group who developed cardiac events had stage II (44\%) and stage IV (56\%) diseases. In our study, as the disease stage increased, cardiotoxicity did not increase. Such a result may have derived from the fact that the number of patients in the cardiotoxicity group is relatively small.

The existence of diseases that cause comorbidities before the transplant (diabetes mellitus, hypertension, obesity, and hyperlipidemia) has been shown as a risk factor for the development of post-transplant cardiotoxicity in various studies $[14,15]$. In our study, it was found statistically that the presence of pre-transplant comorbidity did not make a difference regarding post-transplant cardiac event development [ $p=0.112]$. If patients are selected correctly before transplantation, they may have chronical disease while they do not have complication about these diseases, patients would have lower risks of cardiac events after transplantation.

With the introduction of high doses of cyclophosphamide since the discovery of anthracyclines, there are hundreds of studies related to their cardiotoxic effects [16]. Because the intensity of chemotherapy exposed will increase as the number of cycles increases, we thought that this situation may have an effect on the development of cardiac events after transplantation. In our analysis, we found that the number of cycles was similar in both groups. When we examine the cardiac effects of cyclophosphamide and anthracyclines, it is observed that they cause congestive heart 
failure by causing heart muscle damage in the long term [17]. The reason for the absence of any difference between the two groups in our study may be the fact that we examine the early effects after transplantation. There are some publications showing that a difference is detected when long-term follow-ups are made [16, 17].

It can be estimated that patients with low pretransplant performance may develop more cardiac events after transplant. In our study, 1 patient with KPS was 80, and the others consisted of 90 and 100 patients. In our study, there was no difference between these groups. According to the results of our study, it can be thought that patients with a KPS of 80 and above are safe in terms of cardiac effects in the early post-transplant period.

Many studies using cardiotoxic chemotherapy agents have found that post-procedure cardiac events are common in patients with low LVEF before the procedure. For this reason, it is recommended to evaluate patients with ECO routinely to assess the cardiac functions of the patients before the transplant, since it is a routine cardiology consultation and noninvasive method and is often an affordable, cost effective method [18]. For this purpose, although various parameters are used in echocardiography, LVEF is often calculated. We also prefer patients with LVEF $>50$ for transplantation in our department. When we evaluated the patients in this study in this respect, the median LVEF value of the ejection fraction in the group with cardiotoxicity was calculated as 60 . LVEF value in the control group was 65 and there was a statistically significant difference between the two groups. In a progressive study evaluating patients with lymphoma who used BEAM and CBV chemotherapy regimens as a transplant preparation regimen 2-point decrease in LVEF values was found in the post-transplant patients compared to before the transplant [18]. In this study, we achieved a result that supports the results of many progressive and retrospective studies done on this subject worldwide. These results emphasize the importance of evaluation with echocardiography in the evaluation of patients before transplantation [18-20]. Thus, we eliminate one of the risk factors of a cardiac event likely to develop after transplantation.

DMSO related arythmias can be fatal. One of our patients developed bradycardia during infusion. Therefore, close cardiac monitoring during the trans- plant and especially in patients who are at risk in posttransplant period would be useful for the early detection of cardiac events.

Some studies investigating some anthracycline based chemotherapy protocols have concluded that prophylactic carvedilol and enalapril reduce the development of anthracycline-induced cardiotoxicity [21, $22]$. Although there is no such practice in our routine, it is beneficial to carry out broader prospective studies.

\section{Limitations}

Restrictive factors of our study; the number of patients included in the study and the number of patients developing cardiotoxicity are small.

\section{CONCLUSION}

As a result, the risk factors of the patients should be determined in the pre-transplant period. Their cardiological examination and echocardiographic assessment should be made, and appropriate patients should be directed to the transplant. If cardiac complaints develop after transplantation, we should behave energetically and start treatment immediately after the diagnosis by performing ECG, echocardiography, cardiac marker examinations, so that we can reduce the mortality and morbidity that may develop. Although it is not in our routine, it may be beneficial to use agents such as enalapril and carvedilol in prophylaxis on the condition that it is approved by larger studies.

\section{Authors' Contribution}

Study Conception: SÇ, FY; Study Design: SÇ; Supervision: SÇ, VÖ, FÖ; Funding: N/A; Materials: SÇ, FY; Data Collection and/or Processing: SÇ, FY; Statistical Analysis and/or Data Interpretation: SÇ, FY; Literature Review: SÇ; Manuscript Preparation: SÇ and Critical Review: SÇ.

\section{Conflict of interest}

The authors disclosed no conflict of interest during the preparation or publication of this manuscript.

\section{Financing}

The authors disclosed that they did not receive any grant during conduction or writing of this study. 


\section{Acknowledgement}

This study was presented as a poster at the 27th National Hematology Congress in Antalya on November 1-4, 2017.

\section{REFERENCES}

1. Ferlay J, Shin H, Bray F, Forman D, Mathers C, Parkin DM. Estimates of worldwide burden of cancer in 2008: GLOBOCAN 2008. Int J Cancer 2010;127:2893-917.

2. Muller AM, Ihorst G, Mertelsmann R, Engelhardt M. Epidemiology of non-Hodgkin's lymphoma (NHL): trends, geographic distribution, and etiology. Ann Hematol 2005 84:1-12.

3. Braverman AC, Antin JH, Plappert MT, Cook EF, Lee Rt. Cyclophosphamide cardiotoxicity: a prospective evaluation of new dosing regimens. J Clin Oncol 1991;9:1215-23.

4. Alidina A, Lawrence D, Ford LA, Baer MR, Bambach B, Bernstein $\mathrm{SH}$, et al. Thiotepa-associated cardiomyopathy during blood or marrow transplantation: association with female sex and cardiac risk factors. Biol Blood Marrow Tranplant 1999;5:322-7.

5. Alessandrino EP, Bernasconi P, Caldera D, Colombo A, Bonfichi M, Malcovati L, et al. Adverse events occurring during bone marrow or peripheral blood progenitor cell infusion: analysis of 126 cases. Bone Marrow Transplant 1999;23:533-7.

6. Türk Kardiyoloji Derneği, Koroner Kalp Hastalığından Korunma ve Tedaviye İlişkin Ulusal Kılavuz 1995.

7. Common Terminology Criteria for Adverse Events v4.0 (CTCAE), 2009.

8. Oken MM, Creech RH, Tormey DC, Horton J, Davis TE, McFadden ET, et al. Toxicity and response criteria of the Eastern Cooperative Oncology Group. Am J Clin Oncol 1982;5:649-55. 9. Linch DC, Winfield D, Goldstone AH, Moir D, Hancock B, McMillan A, et al. Dose intensification with autologous bonemarrow transplantation in relapsed and resistent Hodgkin's disease: results of a BNLI randomised trial. Lancet 1993;341:1051-4.

10. Copelan EA. Hematopoietic stem-cell transplantation. N Engl J Med 2006;354:1813-26.

11. Armenian SH, Sun CL, Francisco L, et al. Late congestive heart failure (CHF) following hematopoietic cell transplantation (HCT). 44th Annual Meeting of the American Society of Clinical
Oncology 2008.

12. Murdych T, Weisdorf DJ. Serious cardiac complications during bone marrow transplantation at the University of Minnesota 1977-1997. Bone Marrow Transplant 2001;28:283-7.

13. Hidalgo JD, Krone R, Rich MW, Blum K, Adkins D, Fan M$\mathrm{Y}$, et al, Supraventricular tachyarrhythmias after hematopoietic stem cell transplantation: incidence, risk factors and outcomes. Bone Marrow Transplant 2004;34:615-9.

14. Tichelli A, Bhatia S, Socie G. Cardiac and cardiovascular consequences after haematopoietic stem cell transplantation. $\mathrm{Br}$ J Haematol 2008;142:11-26.

15. Hershman DL, McBride RB, Eisenberger A, Tsai WY, Grann VR, Jacobson JS. Doxorubicin, cardiac risk factors, and cardiac toxicity in elderly patients with diffuse B-cell non-Hodgkin's lymphoma. J Clin Oncol 2008;26:3159-65.

16. Braverman AC, Antin JH, Plappert MT, Cook EF, Lee RT. Cyclophosphamide cardiotoxicity in bone marrow transplantation: a prospective evaluation of new dosing regimens. J Clin Oncol 1991;9:1215-23.

17. Morandi P, Ruffini PA, Benvenuto GM, Raimondi R, Fosser V. Cardiac toxicity of high-dose chemotherapy. Bone Marrow Transplant 2005;35:323-34.

18. Sarzhevskiy V, Kolesnikova D, Melnichenko V, Vakhromeeva M. Cardiotoxicity of high-dose chemotherapy with autologous hematopoietic stem cells transplantation in patients with malignant lymphomas. What is worse - BEAM or CBV? ESMO Ann Oncol 2014;25:423-9.

19. Zver S, Zadnik V, Cernelc P, Kozelj M. Cardiac toxicity of high-dose cyclophosphamide and melphalan in patients with multiple myeloma treated with tandem autologous hematopoietic stem cell transplantation. Int J Hematol 2008;88:227-36.

20. Roziakova L, Bojtarova E, Mistrik M, Dubrava J, Gergel J, Lenkova N, et al. Serial measurements of cardiac biomarkers in patients after allogeneic hematopoietic stem cell transplantation. J Exp Clin Cancer Res 2012;31:13.

21. Cardinale D, Colombo A, Sandri MT, Lamantia G, Colombo N, Civelli M, et al. Prevention of high-dose chemotherapy-induced cardiotoxicity in high-risk patients by angiotensin-converting enzyme inhibition. Circulation 2006;114:2474-81.

22. Kalay N, Basar E, Ozdogru I, Er O, Cetinkaya Y, Dogan A, et al. Protective effects of carvedilol against anthracycline-induced cardiomyopathy. J Am Coll Cardiol 2006;48:2258-62. 